\title{
Sostenibilidad de proyectos de desarrollo con nuevas tecnologías: el caso de la organización de regantes y su sistema de información en Huaral
}

\author{
Juan Fernando Bossio \\ $<$ jfbossio@gmail.com>
}

\begin{abstract}
Resumen
Este artículo utiliza el caso del Sistema de Información Agraria de Huaral (SIA) para analizar la importancia de las organizaciones de base para la sostenibilidad de proyectos de desarrollo que usan nuevas Tecnologías de Información y Comunicación (TIC). El marco teórico es el desarrollo sostenible aplicado al aprovechamiento de TIC en contextos rurales. El caso es estudiado con una mezcla de metodologías cualitativas y cuantitativas. El análisis del caso sostiene la tesis de que el componente social es el más importante para la sostenibilidad final de los proyectos.
\end{abstract}

\section{Introducción}

En América Latina diversos actores han desarrollado proyectos o programas para acortar las desigualdades en el acceso a las nuevas Tecnologías de Información y Comunicación (TIC). Generalmente se asume como beneficioso per se el acceso a Internet (Proenza et al, 2001), lo que lleva a que la mayor parte estos programas sociales se hayan concentrado en facilitar acceso a Internet a grupos de bajos recursos, sin preocuparse por promover un uso apropiado de las tecnologías (Delgadillo et al, 2002; Saravia, 2003).

La mayoría de los proyectos ligados a uso de TIC para el desarrollo enfrentan problemas de sostenibilidad en el tiempo (Davidziuk, 2002). Mucha de la literatura sobre el uso de TIC para el desarrollo entiende la sostenibilidad de los proyectos prácticamente sólo en términos financieros (Proenza, 2001). Sin embargo, desde un sector ligado a la práctica cotidiana del uso de TIC para el desarrollo se afirma que la sostenibilidad, en tanto capacidad de continuar funcionando sin afectar las capacidades de desarrollo, tiene elementos sociales, políticos, tecnológicos y organizacionales además del financiero (Delgadillo et al, 2002; Fukao, 2004; Stoll \& Menou, 2003)

En este artículo nos interesa explorar la importancia de instituciones locales u organizaciones de base en las posibilidades de los proyectos de conseguir sostenibilidad a largo plazo. El caso a analizar será el proyecto Sistema de Información Agraria de Huaral (SIA), que es uno de los proyectos en el Perú que está tratando de mostrar que el acceso a la información puede ser una herramienta de desarrollo y que este acceso puede darse a través de las nuevas TIC. Basaremos el análisis en la evaluación de medio término realizada en febrero y marzo de 2005 (Bossio \& Rocha, 2005).

Las distintas facetas de la sostenibilidad y, especialmente, la sostenibilidad social son discutidas en la siguiente sección luego de ponerlas en el contexto de teorías del desarrollo. En la tercera sección se presenta el contexto general del proyecto. La cuarta relata la historia del proyecto. La siguiente evalúa el proyecto mirando a sus efectos y resultados. Posteriormente analiza de la experiencia a la luz de los conceptos discutidos inicialmente para así poder llegar a conclusiones de utilidad para futuras experiencias. Como anexo se incluye la descripción de la mezcla metodológica utilizada en la investigación y los límites de ésta.

\section{Desarrollo sostenible y sostenibilidad}

Para entender el concepto de sostenibilidad hace falta visitar las teorías alternativas de desarrollo surgidas en respuesta tanto a las teorías de modernización por etapas como a la teoría de la dependencia (Thomas, 2000). Amartya Sen (1999) entiende el desarrollo como un proceso de expansión de la libertad, entendiendo la libertad como el conjunto de capacidades que permiten satisfacer necesidades al ampliar las opciones de la persona, de donde se sigue que el desarrollo estaría ligado a la participación y el empoderamiento. El concepto de desarrollo humano -basado en los postulados de Sen- introducido por las Naciones Unidas incluye cuatro capacidades: "llevar una vida larga y sana, ser reconocible, tener acceso a los recursos necesarios para alcanzar un estándar de vida decente y participar en la vida de la comunidad" (UNDP, 2004: p.127). 
El informe de la Comisión Mundial para el Medio Ambiente y el Desarrollo da origen a la teoría del desarrollo sostenible, al que definen como "el desarrollo que satisface las necesidades del presente, sin comprometer la capacidad para que las futuras generaciones puedan satisfacer sus propias necesidades" (WCED, 1987: p.43). El uso del término "sostenibilidad" por las ONG y agencias internacionales de desarrollo ha ido más allá de consideraciones ambientales y de recursos naturales. Actualmente, este término es usado para describir la capacidad de un proyecto o sus resultados de continuar existiendo o funcionando más allá del fin del financiamiento o las actividades del agente externo (Fukao, 2004). Esta capacidad de seguir existiendo ha sido reducida por muchos actores de desarrollo al componente financiero, de modo que se equipara la evaluación de la sostenibilidad al análisis de flujo de caja. Sin embargo, el desarrollo sostenible y la sostenibilidad suponen la combinación de metas económicas, ecológicas y sociales, como es satisfacer las necesidades básicas, protegiendo el medio ambiente y empoderando a la gente, teniendo a las comunidades locales como actores principales (Holmberg \& Sandbrook, 1992)

La atención de algunos sectores en la sostenibilidad de los proyectos que usan TIC para el desarrollo se ha centrado en el lado financiero de ésta (Proenza, 2001). Sin embargo, otros han definido distintos aspectos de la sostenibilidad: el económico o financiero, el político y legal, el social y cultural, y el tecnológico (Delgadillo et al, 2002; Stoll \& Menou, 2003) a los que Fukao (2004) agrega el organizacional y de recursos humanos. Todos estos aspectos deberían ser considerados con el objetivo de dar continuidad a las acciones y a que éstas no afecten la capacidad de desarrollo futuro.

La sostenibilidad social y cultural está dada por la consideración de las particularidades de los contextos donde se hacen los proyectos y cómo se insertan en ellos convirtiéndose en un 'bien público', tiene que ver con la participación de la comunidad y sus organizaciones y en cuánto se apropian de los proyectos; la política o legal comprende el marco legal o la estabilidad política que permita la continuidad de las acciones de desarrollo; la tecnológica corresponde a la capacidad de actualizar la mezcla de tecnologías seleccionada, y la organizacional está dada por la capacidad de administrar localmente el proyecto y por el desarrollo de recursos humanos locales que permitan su continuidad. La experiencia ha mostrado que tener sostenibilidad social, tecnológica, organizacional y legal sirve para conseguir sostenibilidad financiera, mientras que lo contrario no ha sido observado (Fukao, 2004; Stoll \& Menou, 2003).

Resulta importante analizar cuáles son los plazos en los que la sostenibilidad es juzgada. Cuando se habla en términos financieros, normalmente se tienen proyecciones de corto o mediano plazo. Sin embargo, una actividad financieramente insostenible a corto plazo puede generar sostenibilidad política y social a largo plazo (por ejemplo la prestación de salud pública), mientras que una actividad financieramente sostenible en el corto plazo puede no ser legal (venta de reproducciones ilegales de películas o software). Por ello, es importante considerar entonces que la sostenibilidad tiene metas de largo plazo, pero actividades de corto plazo.

\section{Sostenibilidad del SIA Huaral}

El proyecto SIA Huaral será sostenible en la medida que pueda resolver las necesidades de los agricultores actuales sin hipotecar sus posibilidades de resolver las de los futuros agricultores, es decir que debe tener la capacidad de servir de manera continua en la actualidad sin socavar sus propias capacidades de brindar servicios en el futuro. No solamente se trata de que obtenga recursos financieros que le permitan funcionar hoy sin agotar financiamientos externos, malacostumbrar al mercado, debilitar los ahorros de la institución promotora o recurrir a créditos onerosos; debe construir sostenibilidad en términos sociales, político/legales, tecnológicos y organizacionales.

Este artículo profundiza en la sostenibilidad social y en el papel de la organización de base para conseguirla. Para ello a continuación examinamos el contexto general del proyecto.

\section{Contexto General}

Presentamos a continuación una serie de elementos que sirven para situar el proyecto en su contexto histórico, social y económico, luego describimos la organización con la que se realiza el proyecto y finalmente a los agricultores para los que trabaja el proyecto.

\section{Contexto del proyecto}

El Valle de Huaral se encuentra ubicado en la parte media-baja y baja de la cuenca del río Chancay, tiene un clima desértico cálido y su producción agropecuaria diversificada y continua depende de la irrigación. La agricultura da empleo, de manera directa o indirecta, a la mayor parte de la población económicamente activa de los tres distritos en los que se desarrolla el proyecto (Chancay, Huaral y Aucallama, en la Provincia de Huaral, Departamento de Lima); la ciudad de Lima a unos $90 \mathrm{Km}$. de distancia es el principal mercado para su producción. Dentro de los tres distritos hay varios centros menores con algunas carencias en servicios básicos, infraestructura vial y telecomunicaciones.

Actualmente el 96\% de los agricultores manejan parcelas menores a 10 hectáreas. La Reforma Agraria de 1969 (Matos Mar 1980; Matos Mar 1984) expropió grandes extensiones de tierra y las entregó a cooperativas formadas por sus trabajadores o a comunidades campesinas. Las cooperativas agrarias atravesaron un proceso de parcelación que se 
inició en la década de 1980 y se consolidó en la década de 1990 (Eguren 1988; Fernández y Gonzáles 1990). Dicha fragmentación eliminó las economías de escala y trajo como consecuencia menor rendimiento económico, trabas para la innovación tecnológica, desventaja en la comercialización y menor acceso al crédito formal.

En el Perú los agricultores no suelen tomar en cuenta factores como pronósticos climáticos u oportunidades de negocio para cambiar sus usos productivos o comerciales y persisten con la opción que mejor conocen (Bossio, 2002; Cancino, 2001; INEI, 1996). Aquellos que realizan cambios se apoyan en los familiares, amigos y vecinos como medio de acceder a información comercial y a experiencias productivas, mientras que para asuntos tecnológicos suelen recurrir a los técnicos de las casas comerciales. Los estudios superiores permiten a algunos agricultores poder acercarse a ciertas fuentes de información con más facilidad, pero eso no los lleva a perder las fuentes de información más tradicionales.

\section{La organización de los regantes}

La agricultura de Huaral, como la de los otros valles de la Costa, depende del riego y la organización del sistema de riego siempre ha imbricado su organización social. En tiempos de la Hacienda la administración del agua de riego forzaba la relación de padrinazgo entre hacendados, yanacones y comuneros, mientras que luego de la Reforma Agraria fue necesaria una mayor reglamentación recayendo en el Estado la gestión del recurso (Oré, 1989).

En 1979 entran en vigencia en todo el Perú nuevas organizaciones para la administración del riego que son las Juntas de Usuarios y las Comisiones de Regantes. Las Juntas de Usuarios deben velar por el mantenimiento de la infraestructura de riego, es decir canales y bocatomas, así como infraestructura para conservación de agua. Las Juntas realizan estas tareas con recursos provenientes del cobro de tarifa de agua ${ }^{1}$. En Huaral hay unos 6000 'usuarios de riego' que son los propietarios de los terrenos agrícolas y socios de la Junta ${ }^{2}$. La Junta se divide en 17 Comisiones de Regantes, cada comisión es alimentada por uno de los grandes canales de regadío y normalmente ocupa el territorio de una de las antiguas cooperativas; por ello no tienen similar tamaño o número de socios.

Los sectoristas son funcionarios de la Junta, asignados a diferentes comisiones, que hacen la distribución de agua y la asignación de los turnos de riego, y controlan el buen estado y mantenimiento de la infraestructura de riego. Adicionalmente, dan capacitaciones y cumplían el rol de correo, llevando y trayendo encargos de la Junta a las comisiones y viceversa. Su relación directa con el agricultor les permite estar al tanto de que se está sembrando en el valle, así como de qué problemas se enfrenta durante el ciclo productivo y la comercialización.

Otras organizaciones de los agricultores que se vienen desarrollando son las asociaciones de productores. Estas asociaciones buscan mejorar la productividad a través de capacitación o asesoría técnica. También intentan obtener ventajas comerciales a través de la compra de insumos al por mayor o la venta de productos de forma asociada.

\section{Características de los usuarios o agricultores}

Se puede categorizar a los agricultores de Huaral por sus diferencias en cuánto al origen de la propiedad de la tierra, edad de quien toma las decisiones trascendentes en el fundo y nivel educativo. Además se los puede dividir también de acuerdo a la zona en la que está su parcela (parte baja, media o alta del Valle).

En cuanto a la propiedad de la tierra, la primera diferencia se da entre los propietarios y los arrendadores; estos últimos pueden ser residentes permanentes en el valle, emigrantes periódicos u ocasionales. Hay cuatro tipos básicos de origen de la propiedad: por compra en lotización o irrigación antes de la reforma agraria, por ser beneficiario de la reforma agraria (yanacones o peones ${ }^{3}$ ), por herencia o por haber comprado el terreno después del proceso de parcelación a alguno de los tres tipos anteriores o en una nueva irrigación. Esta diferencia tiene correlato en la experiencia empresarial de los agricultores, ya que los peones beneficiarios de la reforma agraria formaron cooperativas hasta los ochentas o noventas del siglo pasado mientras que los demás han tenido más experiencia en el manejo de parcelas privadas.

En cuanto a la edad, si bien la mayor parte de la población rural del valle de Huaral se encuentra entre los quince y cuarenta años, la mayoría de los agricultores titulares de las parcelas ('usuarios de riego') son mayores de 50 años (59 años fue la edad promedio de los usuarios encuestados), lo que hace difícil la apropiación de nuevas tecnologías y prácticas agrícolas. En cuanto a nivel educativo, la inmensa mayoría de los agricultores sabe leer y escribir $-93.98 \%$ según el Censo Agropecuario de 1994 (INEI 1996)-, y existen algunos agricultores que tienen formación superior técnica o profesional.

1 Las juntas no cobran por el agua sino por el uso de la infraestructura que permite tener acceso al agua. El agua de riego es un bien público; si ellos cobraran por el agua abrirían la puerta a la privatización de este recurso, idea a la cual se oponen. Pero esta posición lleva a que no puedan impedir el acceso al agua por falta de pago.

2 Ni el peón agrícola ni aquellos que alquilan tierras son 'usuarios de riego', sólo lo son los propietarios de los terrenos inscritos en las comisiones.

3 Los yanacones o yanaconas arrendaban al hacendado las tierras que usaban, mientras que los peones eran obreros asalariados. En muchos casos los yanacones recibieron pequeñas extensiones de tierra antes de que la reforma agraria llegara a Huaral, mientras que los peones fueron organizados en cooperativas agrarias. 


\section{Historia del proyecto}

En esta sección se describe la evolución del proyecto desarrollado por el Centro Peruano de Estudios Sociales (CEPES) y como éste se va modificando en respuesta al ambiente. Primero se describe el desarrollo de la idea de proyecto y luego la interacción con otros actores y contrapartes; después se esboza una cronología de la implementación; y finalmente se presenta los retos actuales del proyecto. Esta sección fue construida a partir de los testimonios de varios de los principales actores del proyecto como Ignacio Cancino (IC), Maicu Alvarado (MA), Héctor Salvador y Marcial Vega (MV), así como otros directivos de comisiones, agricultores y administradores de telecentros.

\section{Dándole forma a una idea}

En el año 2000, IC de CEPES visitó la Municipalidad Distrital de Aucallama para invitarla a participar en un proyecto de uso de computadoras e Internet para mejorar su funcionamiento interno y servicios. La Municipalidad no le prestó atención, pero el regidor MV - presidente de una comisión de regantes- sugirió que el proyecto se realizara con la Junta de Usuarios.

Desde inicios de 2001 la Junta de Usuarios - con MV de presidente- y CEPES desarrollan la idea de proyecto. La idea inicial era dotar de una computadora a cada comisión de regantes y conectarla a Internet con antenas satelitales con el objetivo de mejorar la administración de la comisión y para que los agricultores accedieran a información agraria a través de la persona que administraría el uso de la computadora. La preocupación por la sostenibilidad financiera mostrada por potenciales donantes desnudó dos problemas: el alto costo de la opción tecnológica elegida y la ausencia de fuentes de ingresos que permitieran solventar financieramente el sistema luego de terminado el financiamiento externo.

Una consultoría de la Red Científica Peruana (RCP) recomendó utilizar radio-enlace para interconectar los locales de las comisiones abaratando el costo de conexión y vender acceso a Internet a colegios para generar fondos, idea que luego evoluciona hacia proveer acceso público. La alternativa de brindar servicios al público consigue el inmediato apoyo de los miembros de la Junta y las comisiones a quienes interesó la posibilidad de contribuir con el desarrollo local y la mejora de la calidad educativa.

Desde ese momento la idea del proyecto fue la de un sistema de información agraria, que usa tecnología de radioenlace para interconectar varios puntos en el valle y que tiene por objetivos contribuir con la apropiación de TIC por agricultores y población en general, mejorar la administración de la organización de los regantes, permitir el acceso de agricultores a información útil y apropiada, y que debía financiarse a través de la prestación de servicios de telecomunicaciones.

\section{Los actores institucionales relacionados al proyecto}

Este proyecto no ha sido nunca impulsado solamente por CEPES sino que siempre ha contado con el apoyo de la Junta de Regantes. La relación con otras organizaciones o instituciones también ha dado forma al proyecto. Acá agrupamos por sectores a las instituciones con las que el proyecto se ha relacionado.

El compromiso institucional de la Junta con el proyecto ha sido permanente. Fue una asamblea la que tomó la decisión de participar del proyecto desde 2001 y otra la de comprar 12 computadoras como aporte económico de la Junta en 2002. Además, de las 12 comisiones que tienen telecentros -con entre 3 y 5 máquinas cada uno-, una remodeló y amplió su local, mientras que otras 10 debieron construir o alquilar local a raíz del proyecto. Otras organizaciones de agricultores que no fueron consideradas en el planteamiento inicial -como las asociaciones de productores- ahora están siendo incorporadas a la propuesta.

El proyecto se ha relacionado con tres sectores dentro del aparato estatal: telecomunicaciones, agricultura y educación. Desde 2001 se buscó financiamiento del Fondo de Inversión en Telecomunicaciones (FITEL), el que demoró tres años en llegar, primero por trabas legales (no estaba permitido el uso de radio enlace) y después burocráticas (idas y venidas para la aprobación del directorio de OSIPTEL - del que dependía el FITEL- y del Ministerio de Transportes y Comunicaciones). Las instituciones del sector agricultura -tales como el Instituto Nacional de Investigación Agraria (INIA), el Servicio Nacional de Sanidad Animal (SENASA) y la Agencia Agraria del Ministerio de Agricultura- fueron consideradas como socios claves para la propuesta por que manejan grandes cantidades de información, potencialmente útil para los agricultores, pero no tenían los medios para hacerla llegar adecuadamente. Desde mediados de 2001 se buscó incorporar al sector educación al proyecto dado que, por un lado los agricultores de la Junta consideraban que sus hijos o nietos podían beneficiarse del proyecto, y por el otro, la consultoría de la RCP recomendó que el sector educación fuera un 'cliente institucional'; en tal sentido la Unidad de Gestión Educativa Local (UGEL) -encargada del sector educativo en Huaral- firmó un contrato de servicio con el proyecto en noviembre de 2001, pero reiteradas trabas burocráticas llevaron a que se dejara de lado la relación con el sector educación a mediados de 2003.

Desde el principio se buscó incluir a las casas comerciales como proveedores de información de precios de insumos, pero estas empresas no participan pues no ven beneficio en hacerlo. En 2005 los laboratorios fueron 
identificados como fuente de información técnica y se hicieron contactos que permiten ahora que estos proveedores de insumos entreguen información al sistema.

\section{La implementación del proyecto}

Desde 2001 CEPES ha desarrollado actividades ligadas a este proyecto usando financiamientos de distintos tamaños, fuentes, duraciones y momentos de inicio, así como financiamiento del mismo CEPES. En febrero 2001 se realiza un diagnóstico de necesidades de información (Cancino, 2001), financiado por la Cooperación Técnica Alemana (GTZ), que describe de manera general las necesidades de información de los pobladores, su relación con el calendario agrícola y las fuentes de información usadas para obtener esa información. En noviembre de 2002 empieza el diseño del sistema -basado en el diagnóstico inicial y trabajo de grupo con actores interesados- y el desarrollo de la plataforma de software para el sistema.

En 2003 CEPES consigue financiamientos del sector agricultura: en mayo de la Dirección General de Información Agraria (DGIA) del Ministerio de Agricultura para capacitar a agricultores en el uso de computadoras e Internet y en octubre del Proyecto INCAGRO (Innovación y Competitividad en el Agro) para el desarrollo y carga de la plataforma de información del Sistema de Información Agraria. En agosto de 2003 empieza la capacitación de 280 agricultores, ésta es la primera actividad de campo de gran escala con la población beneficiaria y marca en nuestra opinión el inicio del proyecto. En ese momento aún no había Internet en las comisiones ni información accesible en la Web, pero ya se había capacitado usuarios e intermediarios, empezado a reunir contenidos, desarrollado lazos institucionales, e invitado a la participación de la población, los que son componentes fundamentales de un proyecto de desarrollo que tiene a Internet como herramienta.

En 2004 se desarrolla la plataforma de contenidos (http://www.huaral.org); se prosigue la capacitación y se selecciona a los administradores de los telecentros; se realiza un censo agropecuario que sirve para alimentar de información al sistema; se realiza la mayor parte de los desarrollos informáticos y, finalmente desde septiembre de 2004 -cuando se ejecuta el financiamiento de FITEL- empieza la puesta en funcionamiento de Internet en las comisiones. De haber tenido mayor flexibilidad respecto a la alternativa tecnológica elegida se podría haber iniciado antes el proyecto de sistema de información -aún sin puntos de acceso a Internet- en vez de haber esperado por el financiamiento de FITEL.

\section{Situación del proyecto}

En Marzo de 2005, cuando realizamos la evaluación, CEPES buscaba darle estructura y continuidad a lo implementado por el proyecto, mientras avanzaba en el proceso de transferencia. La Junta ya se había consolidado como el principal proveedor de información del sistema y los sectoristas de la Junta empezaron luego a ingresar información. Se buscaba incrementar la participación de otras instituciones locales o de instituciones nacionales ligadas al agro, actividad en la que se ha avanzado desde entonces, especialmente por el reconocimiento que está teniendo la Junta en cuanto al tema de sistemas de información. El equipo de informáticos de CEPES es aún necesario en Huaral ya que no han llegado a transferir todas sus responsabilidades al personal local, en un proceso que avanza pero que tiene ciertos cuellos de botella; por ejemplo, se ha transferido capacidades al personal local y de la Junta para actualizar información y crear páginas Web mientras CEPES investiga nuevas aplicaciones que para su desarrollo requieren de profesionales con los que no cuenta la Junta.

La incorporación de la atención al público tuvo la finalidad de darle sostenibilidad financiera al proyecto a través del cobro por acceso a Internet y servicios informáticos. Es claro ahora que ésa no puede ser la única forma de obtener recursos financieros, por ello CEPES y los dirigentes están analizando las opciones que tiene para generar fondos. La visión de CEPES y la Junta es que el sistema es un todo y su sostenibilidad financiera responde al todo, pero la percepción desde algunas comisiones es que cada telecentro debe velar por su propia sostenibilidad financiera. Sin embargo, hay telecentros con altos ingresos y otros con ingresos muy bajos, lo que se explica, en unos casos, por la presencia o no de competencia en la prestación del servicio de Internet y, en otros, por el tamaño de los centros poblados atendidos. Es claro que algunos telecentros deberán ser financiados ya que no podrán sostenerse financieramente por la venta de acceso a Internet.

Por el momento, la Junta de Usuarios cubre el costo de recursos humanos de algunos telecentros mientras que las comisiones cubren los costos por servicios y otros gastos. En cuánto al sistema de información por la página Web, mucha de la alimentación de información se ha incorporado al funcionamiento de las instituciones, mientras que el mantenimiento y desarrollo informático sigue en manos de CEPES.

\section{Evaluación del proyecto}

Esta sección presenta los efectos que se puede identificar desde el inicio de acciones conjuntas entre CEPES y la Junta en 2001, así como algunos resultados del proyecto hasta marzo de 2005. La mayor parte de los efectos presentados tienen que ver con el objetivo de mejorar la organización de regantes y describen el fortalecimiento 
institucional, mejora en la eficiencia comunicativa y administrativa, también se esbozan efectos en acceso a las telecomunicaciones. Finalmente se describen una serie de resultados ligados a la participación social en el proyecto.

\section{Fortalecimiento Institucional}

Las entrevistas a directivos de la Junta y las comisiones así como a otros informantes claves dan cuenta de un fortalecimiento de la Junta desde enero de 2001. Este proceso se muestra, por ejemplo, en que la Junta de Usuarios tiene un nuevo local de $1650 \mathrm{Mt}$; ha duplicado el almacenamiento de agua en las lagunas que proveen agua a Huaral; y ha disminuido radicalmente la morosidad en el pago de la tarifa de agua. El mismo proyecto SIA es una muestra de dicho fortalecimiento en tanto otras Juntas de Usuarios quieren realizar réplicas del proyecto (recientemente lo han hecho las juntas de Jequetepeque, en Cajamarca, y Moche, en La Libertad, ambas con asesoría de la Junta de Usuarios y de CEPES). Según Julio San Román, vicepresidente de la Junta de Usuarios, ésta se ha convertido "en la organización más importante del valle de Huaral" por el reconocimiento que tiene tanto a nivel local como nacional, tomando en los últimos años un lugar de liderazgo a nivel nacional entre otras organizaciones de regantes. La voz de la Junta se hace escuchar en medios de comunicación y directorios de organizaciones gubernamentales. Según Maicu Alvarado, "ahora Marcial (Vega) se reúne con el Ministro, y el INIA y el SENASA lo buscan, eso no pasaba con los anteriores presidentes de Junta". Además, la Junta ha recibido visitas de instituciones nacionales e internacionales a propósito del proyecto. Todo esto contribuye a mejorar la autoestima de los miembros y dirigentes de la organización de regantes y fortalece la identificación de los miembros con su organización.

A su vez, las comisiones también se han fortalecido. Las que tienen telecentros cuentan ahora con local, muchas no tenían personal permanente y ahora tienen al administrador del telecentro que suele cumplir funciones administrativas. Los locales de los telecentros se han vuelto puntos de referencia a donde los usuarios pueden acercarse con sus quejas o sugerencias, dejar mensajes, hacer trámites, recibir comunicados e información de la Junta, buscar al sectorista o hacer reuniones, con lo que aumenta la participación social. En resumen, el proyecto ha servido para motivar la institucionalización de las organizaciones de base de los regantes, permitiendo que las comisiones también tomen un rol de servicio a toda la comunidad y no sólo a los usuarios de riego.

No se puede atribuir todo el fortalecimiento institucional al proyecto, pero para todos los entrevistados es indudable que la gestión concertada del proyecto y la difusión que éste ha tenido han sido condición necesaria de dicho proceso.

\section{Mejora de los flujos comunicación en la organización de regantes}

La capacidad de comunicación dentro de la Junta (con y entre las comisiones) y hacia fuera se ha incrementado gracias a la instalación de Internet y los teléfonos internos en las comisiones, luego convertidos en anexos que permiten hacer llamadas al exterior desde comunidades donde no hay servicio de telefonía. Antes del proyecto no había comunicación directa entre las comisiones y la Junta, y la interacción entre las comisiones era mínima. El acceso a teléfono era muy limitado (sólo dos comisiones tenían teléfono) y un mensaje podía demorar 2 días en llegar mientras era llevado por el sectorista, lo que muestra en cuanto ha mejorado la comunicación entre la Junta y las Comisiones de Regantes como efecto directo del proyecto. También ha disminuido sustancialmente el gasto en comunicación de la Junta, porque antes había que llamar al teléfono o a celular, y ahora la comunicación se hace por interno o Chat. La Junta no tenía acceso a Internet antes del proyecto y para consultar el correo electrónico usaba cabinas públicas (cybercafé). Tal como hemos resaltado, al hablar del fortalecimiento institucional, la Junta tiene ahora la posibilidad de interactuar con otros actores a nivel local, nacional e internacional. Antes del proyecto no sólo no tenía los contactos sino tampoco los medios para comunicarse más allá de Huaral. El tener una página de Internet ha permitido que la Junta sea contactada y el correo electrónico le permite comunicarse con interlocutores fuera de Huaral.

La disponibilidad de estas herramientas tecnológicas ha mejorado ciertos flujos de información y comunicación mientras que otros no se han alterado. Entre los flujos que han cambiado tenemos la comunicación con el sectorista especialmente cuando es compartido por más de una comisión-, la comunicación con instituciones externas, la emisión de comunicados de prensa y la consulta de estado de pago de tarifa de agua. Mientras que no han cambiado otros tipos de comunicación como las invitaciones, notificaciones, comunicados formales, convocatoria a asamblea, reclamos y denuncias, los que siguen haciéndose como antes. El análisis muestra que cuando la variable urgencia es de mayor importancia que la variable formalidad entonces el uso de nuevas tecnologías se abre paso. 


\section{Comunicación Urgente}

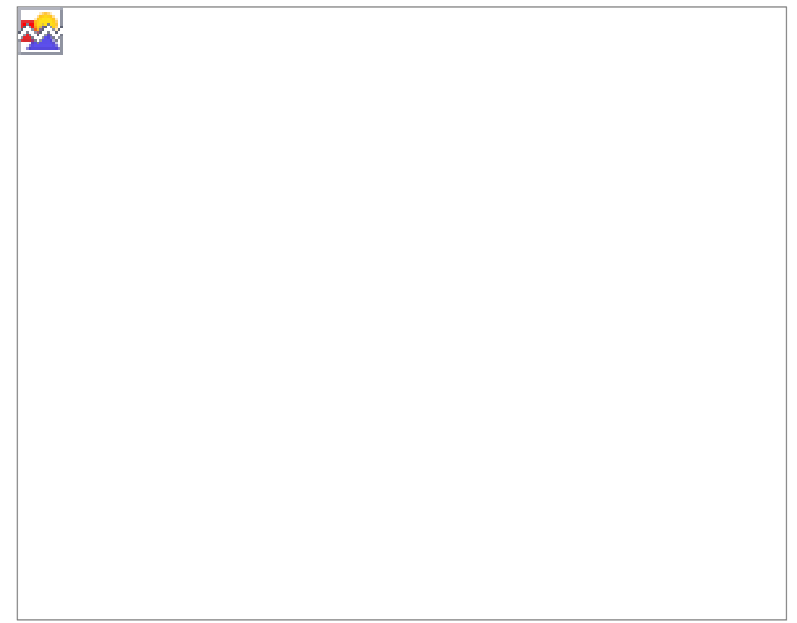

\section{Comunicación Formal}

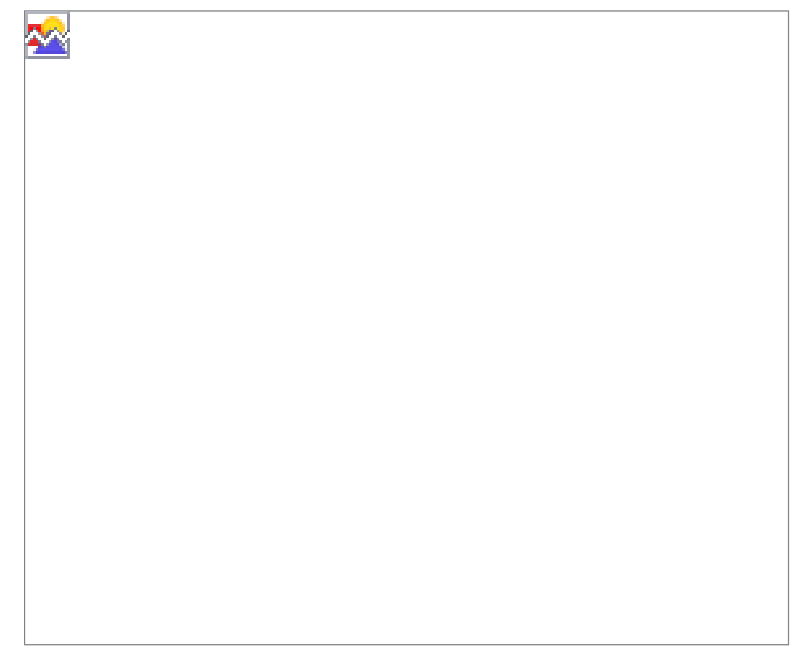

Sabemos que los flujos de información y comunicación tienen hondas raíces culturales por haberse acomodado a condiciones sociales, económicas, políticas y/o ambientales (Bossio 2002), por lo que la simple disponibilidad de tecnología no siempre los transforma. Los agricultores esperan ver firma y sello en el tipo de comunicaciones que no han cambiado. Por ejemplo, se siente más obligación de asistir a una asamblea si se recibió una carta que cuando se escuchó un comunicado por radio o se recibió invitación verbal. Lograr cambios culturales que aminoren el valor que se da a los 'documentos' tomará tiempo y tal vez haya que esperar a la próxima generación de agricultores; mientras tanto se podrían buscar formas de acomodar el uso de herramientas como firma digital y digitalización de documentos para aprovechar las tecnologías disponibles y mejorar la eficiencia de ciertos flujos de información y comunicación.

\section{Mejora en el funcionamiento administrativo de la Junta y las comisiones}

Los sectoristas consideran que ahora tienen facilidades que les han permitido incrementar la eficiencia de su trabajo. Diversas tareas administrativas que antes debían hacerse en Huaral -como la digitación de documentos- pueden hacerse ahora en las comisiones. El acceso a Internet y a las computadoras les permite diseñar mejor los roles de riego, acceder a información y comunicarse con los otros sectoristas de una forma nueva: el correo electrónico.

Los problemas de cobranza se están solucionando gracias a que los usuarios tienen la posibilidad de obtener inmediatamente información sobre sus pagos pendientes desde los locales de cada comisión, para lo que antes debían ir a Huaral. Esto mejora el reconocimiento y valoración de la organización por sus miembros.

Las comisiones de regantes están publicando sus propias páginas Web. La elaboración de estas páginas ha servido para relacionar más al telecentro con su respectiva comisión. En opinión de los administradores, esta publicación ayuda a una mayor vigilancia de los dirigentes por los miembros de la comisión, especialmente por aquellos que viven en Lima o en otras zonas del valle, lo que redunda en una mejora en la administración de la organización de base.

\section{Mayor acceso a servicios de telecomunicación para la población en general}

En la mayor parte de los lugares donde se ha instalado telecentros no había acceso a Internet antes del proyecto, acceder al servicio suponía un viaje a la ciudad de Huaral, con un costo extra en tiempo y dinero. Si bien aún no se consigue que muchos agricultores utilicen los telecentros para buscar información agraria, es claro que el conocer al responsable del telecentro ayuda a que quienes no saben usar la computadora se acerquen a hacerlo. Por otro lado, el servicio de Internet le ha dado a la juventud de los centros poblados sede de las comisiones una posibilidad que antes no tenía. Adicionalmente el proyecto ha ayudado a ampliar la cobertura telefónica dotando del servicio a 5 poblados que no contaban ni con un teléfono público (Caqui, Huayán, Chancay Bajo, Chancay Alto, Cuyo).

Como efecto indirecto (y no esperado) se ha promovido el negocio privado de acceso a Internet ya que en 5 localidades -de las 10 en las que no había Internet antes de que empezara el proyecto- ahora hay empresarios privados que han instalado cabinas públicas, en algunos casos a raíz de la promoción y capacitación, en otros casos inmediatamente después de la instalación de los telecentros y debido a su gran demanda.

\section{Apropiación social}


La evaluación de algunos de los resultados de las actividades del proyecto son importantes para conocer el grado en el cuál el proyecto está o no siendo apropiado por la comunidad. Estos resultados incluyen el nivel de conocimiento del sistema, la calidad de la información en el sistema y la participación de otras instituciones.

\subsection{Nivel de conocimiento de los telecentros y el SIA por los agricultores}

Casi el 70\% de los usuarios de riego sabía de la existencia de computadoras conectadas a Internet en el local de su Comisión. De éstos, la gran mayoría (79.4\%) se enteraron al visitar el local de la comisión para hacer trámites o asistir a reuniones; le sigue el grupo que se enteró por amigos vecinos y familiares (14.7\%). Sin embargo, un $90 \%$ no las había usado, la mayoría porque no sabía usarlas $(55.3 \%)$ o por falta de tiempo $(20.2 \%)$. El problema no era que no se sabía que existían los telecentros, sino que no se sabía que podían ser útiles y que se tiene derecho a usarlos. Luego de la evaluación la asistencia de los agricultores se incrementó gracias a actividades de promoción de la utilidad de Internet y de capacitación.

Sólo el 32.8\% de los usuarios de los telecentros había visitado la página Web del SIA. La mayor parte de los que la usaban son personas mayores de 30 años que son agricultores, empleados o amas de casa. Es esperable que el aumento de la asistencia de agricultores esté significando un aumento en el uso del SIA. El hecho de que la mayor parte de la información presente en el SIA venga de la propia Junta de Usuarios contribuye a que sea consultada por la confianza que existe en la fuente de información.

\subsection{Información en el sistema}

La definición de los módulos del sistema e identificación de los oferentes de información fue hecha en el diagnóstico de necesidades de información (Cancino, 2001). El diseño de cada módulo fue producto de un trabajo en equipo con la participación de los actores directamente interesados. Se diseñaron bases de datos para la información factual (precios, volúmenes de agua, áreas cultivadas) a ser provista por distintos actores. En cuanto a información temática, especialmente la información técnica, las prioridades no se definieron en base a diagnósticos sino por la oferta de información (por ejemplo un video de Bayer) o por la demanda de grupos concretos (por ejemplo un boletín dirigido a los ganaderos). La información de noticias no estaba originalmente planeada y surgió para darle cotidianeidad a la página.

El 70\% de los que lo usan la página del SIA la considera útil o muy útil. La información presentada en el sistema es considerada comprensible o muy comprensible por el 97\% de estos Sin embargo, debemos relativizar esta calificación considerando que tan sólo un tercio de los usuarios de los telecentros usan el SIA y menos del 10\% de los agricultores usa las computadoras de los telecentros.

\subsection{Participación de diversos actores en la alimentación del sistema}

La alimentación del sistema se está descentralizando cada vez más. Cuando se hizo la evaluación la mayor parte de la información era recogida, procesada y colocada por CEPES, ahora la Junta de Usuarios es el principal proveedor de información en el sistema y realiza todas las tareas autónomamente, mientras que otros socios entregan a CEPES o la Junta información procesada.

Son varias las instituciones que alimentan de información al sistema: la Junta registra pagos de tarifa, noticias y volúmenes de agua (en conjunto con la Administración Técnica de Riego del Ministerio de Agricultura), SENASA (Servicio Nacional de Sanidad Agraria) provee información técnica y da acceso a su base de datos de plagas, la DGIA da información de precios y producción en otros mercados y la Estación Experimental Agraria Donoso del INIA (Instituto Nacional de Investigación Agraria) provee información sobre sus servicios y facilita información técnica. Las asociaciones de productores colocan información sobre sus actividades. Algunas empresas privadas facilitan información técnica. Es relevante que las propias comisiones, a través de equipos formados por el administrador del telecentro y otros jóvenes, están ingresando información en el sistema sobre sus localidades.

\section{Análisis}

Como decíamos al principio, la sostenibilidad tiene también otras facetas que nos llevan a hablar de sostenibilidad política o legal, sostenibilidad tecnológica, sostenibilidad organizacional y sostenibilidad social y cultural, además de la financiera.

En términos legales, hacer sostenible el proyecto en Huaral implica cumplir con los reglamentos y las leyes establecidos y tener la flexibilidad para acomodarse a cambios razonables para evitar cierres u otras acciones judiciales. La sostenibilidad tecnológica supone que las plataformas de hardware y software del sistema tengan la posibilidad de acomodarse a cambios en el ambiente (social y físico) y que puedan expandirse, de lo contrario las soluciones tecnológicas de hoy se convertirían en las trabas de mañana. En el sentido organizacional, se debe formar los recursos 
humanos locales que el sistema requiere para poder seguir funcionando luego de que los técnicos de CEPES se retiren. Por último, para que el sistema de información agraria de Huaral sea socialmente sostenible debe ser asumido como propio por quienes van a usarlo: los agricultores y su organización, llegando a convertirse en un bien público.

La participación de las organizaciones de base es pieza fundamental de la misma definición de la sostenibilidad social y cultural. Nuestra intención en este artículo es mostrar cuán importante es también para conseguir las otras facetas de la sostenibilidad, así como fortalecer la tesis de que obtener sostenibilidad social sirve para obtener sostenibilidad financiera y no a la inversa.

\section{El papel de la Junta de Usuarios en la sostenibilidad}

Entre aquellos que conocen de la existencia del proyecto o de los telecentros la mayoría lo supieron a través de la Junta. Al hacer propio el proyecto de sistema de información la Junta ha transmitido al agricultor confianza en el proyecto, en el sistema de información y en la información que éste contiene. Es gracias a esta confianza que centenares de agricultores e hijos de agricultores se acercaron a las capacitaciones iniciales y también por esta confianza se acercan ahora a capacitarse en los telecentros. La confiabilidad de la fuente es una de las condiciones para que la información sea apropiada (Bossio, 2002); por ello se debe a la Junta el que en tan poco tiempo el sistema de información esté siendo consultado por los agricultores.

La Junta de Usuarios no era una institución muy fuerte en 2001 y es claro que el proyecto ha contribuido con su fortalecimiento, pero también es cierto que la institucionalidad de la Junta ha contribuido fuertemente a facilitar al proyecto ciertas relaciones con instituciones estatales y privadas. Por ejemplo, ciertos productores de información pueden tener más disposición a dar recursos de información a una organización de agricultores que a una ONG, ya sea porque ven a los agricultores como clientes -en caso de ser empresas privadas- o como beneficiarios de sus acciones en caso de ser organismos estatales.

Así como las comisiones de regantes se han fortalecido debido al proyecto, también el proyecto se hace más sostenible por la participación de las comisiones de regantes que son las organizaciones de base con las que el agricultor se relaciona directa y cotidianamente. Desde las comisiones se obtiene información y se desarrollan relaciones con otras organizaciones de base o instituciones a nivel local, como colegios o comedores populares. Gracias a esto se amplía el reconocimiento del proyecto por mayores sectores de la sociedad a nivel local. Las comisiones son también la mejor plataforma para promover la participación social en el proyecto.

La Junta fue también importante en las actividades de influencia en política pública para obtener las modificaciones a la reglamentación necesarias para permitir el radio-enlace entre los telecentros de las comisiones, antes sólo permitidos entre oficinas de una misma empresa o institución.

En cuanto al desarrollo de capacidades de personal local, la Junta seleccionó a quienes asistirían a las capacitaciones y es la que ahora paga a los administradores y da espacio de trabajo a los voluntarios de los telecentros. La Junta entiende que apoyar el desarrollo de capacidades del personal local es una buena inversión; por ejemplo, en noviembre de 2005 apoyó la participación de 8 administradores en el 3er Encuentro Nacional de Telecentros realizado en Arequipa.

El diseño del sistema ha sido apoyado por la Junta desde la convocatoria a las reuniones para el diagnóstico inicial y así como en las consultas a los múltiples actores que iban a proveer y usar información. De tal forma ayuda a que el diseño sea apropiado a las necesidades, intereses y capacidades de los agricultores.

La Junta de Usuarios sostiene económicamente la provisión de servicios -mediante el pago de los administradoresen las comisiones dónde el alquiler de acceso a Internet no genera suficientes fondos.

Al ser promovido por la Junta, el proyecto está también imbuido de respeto y consideración por la cultura local, evitando choques dramáticos innecesarios. Por ejemplo, se promueve que Internet pueda facilitar y acelerar ciertos flujos de información pero no se fuerza la informatización de todos los posibles procesos ya que algunos pueden tener otras funciones sociales.

Por último, el firme apoyo de la directiva de la Junta ha ayudado a la introducción de TIC en sus procesos administrativos mejorando la atención a los agricultores. El que se le asigne nuevas tareas a los sectoristas es positivo ya que de lo contrario se afectaría su rol social sin darle nada a cambio ya que actualmente han dejado su rol de correo informal.

\section{SIA Huaral: el proyecto de la Junta de Usuarios}

Se están dando pasos para una transferencia por etapas del sistema. Se empieza por aquellas funciones que los recursos humanos de la Junta pueden manejar mientras se desarrollan las capacidades locales para poder transferir conocimientos tecnológicos más complejos. 
Desde 2001 siempre se ha dicho que el Sistema de Información Agraria era un proyecto de CEPES y la Junta de Usuarios. Sin embargo, en los primeros años se veía como un proyecto que CEPES hacía para la Junta. Ahora que la Junta es el principal proveedor de información, que administra y sostiene económicamente los telecentros, y que ya no sólo recibe visitas sino que es convocada a hablar del proyecto, ya se puede decir que el SIA es "un proyecto de la Junta que contó con el apoyo del CEPES".

Desde el inicio, el proyecto tuvo como aliado al actual presidente de la Junta (MV) y contó con el apoyo entusiasta de otros dirigentes. Próximamente se elegirá una nueva directiva, ésta podrá determinar algunos cambios en el proyecto: hay quienes lo quieren más rentable, hay otros que quieren orientarlo a atender las necesidades del agro más moderno y de exportación, puede haber quienes quieran hacerlo exclusivamente agrario y otros que en cambio quieran hacerlo más amplio e incluir las necesidades de otros actores no agrarios como el sector educación o el de salud. Pero no hay nadie, entre las decenas de dirigentes y agricultores entrevistados, que considere cerrar el proyecto y que no lo vea como un logro, un reto y una oportunidad.

\section{Conclusiones}

Una de las principales fortalezas del proyecto es que éste ha sido asumido como propio por la organización de regantes, esto le da sostenibilidad social porque los telecentros se vuelven un bien público obtenido por la población y no un regalo dejado allí por una financiera internacional. Quienes desarrollan el proyecto no son extraños, ciertamente hay técnicos venidos de fuera de Huaral, pero la participación de la Junta y el reclutamiento de jóvenes locales para trabajar en los telecentros permiten que los agricultores de Huaral desarrollen proximidad y confianza.

Esta confianza hace más fácil capacitar a agricultores y conseguir que usen nuevos recursos de información a través de nuevas herramientas. En este caso la sostenibilidad social contribuye con la sostenibilidad tecnológica y organizacional ya que se están desarrollando recursos humanos capaces de asumir la conducción del proyecto.

También se ha visto como la sostenibilidad social contribuye con la sostenibilidad legal por las gestiones respecto al uso de radio-enlace. Así mismo, la sostenibilidad social del proyecto en la Junta permite que los telecentros no rentables sigan existiendo, es decir que les da la esperada sostenibilidad financiera.

La sostenibilidad no es algo que se consiga de una vez, sino algo que se construye de manera continua, algo en lo que se debe seguir trabajando. Creemos que basar la sostenibilidad social del proyecto en una sola organización de base puede ser peligroso. Además, si el proyecto salta alguna vez de ser un proyecto de desarrollo agrario a ser uno de desarrollo rural deberá ampliar el espectro de organizaciones e instituciones con las que trabaja. Por otro lado, el proyecto debe también desarrollar sostenibilidad a nivel local. Esta sostenibilidad -que puede expresarse en la defensa del telecentro por la comunidad en caso de que sea necesario- debe ganarse en cada comisión y para ello los telecentros deben relacionarse con otras instituciones u organizaciones locales.

Consideramos demostrado que la organización de base ha sido fundamental en darle sostenibilidad a esta experiencia y esperamos que esta lección pueda ser usada y acomodada a otras experiencias y proyectos.

\section{Bibliografía}

Bauer, M. y B. Aarts (2000). Corpus construction: a principle for qualitative data collection. En: Qualitative researching with text, image and sound: a practical handbook for social research / M. Bauer and G. Gaskell (pp. 19-37). London: SAGE.

Bossio, J. (2002). Flujos de Información y Comunicación en contextos rurales: punto de partida para intervenciones en Tecnologías de Información y Comunicación. En: Perú, el problema agrario en debate, SEPIA IX / Manuel Pulgar Vidal, Eduardo Zegarra y Jaime Urrutia, eds. (pp. 662-687). Lima: SEPIA, CIES, CARE, OXFAM.

Bossio, J. y J. Rocha (2005). La organización de regantes y el acceso a información en Huaral: apropiación social de nuevas tecnologías: Evaluación de medio término del proyecto Sistema de Información Agraria de Huaral. Reporte no publicado entregado a CEPES, Lima.

Cancino, I. (2001). Determinación de Necesidades Específicas de Información Agraria e Identificación de los Sistemas de Información del Valle de Huaral. Reporte no publicado entregado a CEPES, Lima.

Davidziuk, M. A. (2002) Las TIC como instrumento de inclusión comunitaria y desarrollo social: el caso del Proyecto CTC. Tesina de grado no publicada, Universidad de Buenos Aires, Facultad de Ciencias Sociales.

Delgadillo, K., R. Gómez y K. Stoll (2002). Telecentros... ¿para qué?: lecciones sobre telecentros comunitarios en América Latina y el Caribe. Ottawa: IDRC.

Eguren, Fernando (1988). Revisión y balance de los estudios sobre restructuración de empresas agrarias asociativas. En: SEPIA II: El problema agrario en debate. Lima: SEPIA: 
Fernández, Ángel y Alberto Gonzáles; eds. (1990) La reforma agraria peruana, 20 años después. Chiclayo: Centro de Estudios Sociales Solidaridad.

Fukao, T. (2004). What are the key factors for the rural telecentre's sustainability? a case study of a rural town in Mongolia. Unpublished MSc dissertation, London School of Economics.

Holmberg, J. y R. Sandbrook (1992). Sustainable development: what is to be done? In: Policies for a small planet / J. Holmberg. London: Earthscan.

INEI (1996). Censo Nacional Agropecuario, III - 1994. Resultados Definitivos. Perú. Lima: INEI.

Matos Mar, José (1980). La reforma agraria en el Perú. Lima: IEP.

Matos Mar, José (1984). Reforma agraria: logros y contradicciones 1969-1979. 2da ed. Lima: IEP.

Oré, María Teresa (1989). Riego y Organización: evolución histórica y experiencias actuales en el Perú. Lima, ITDG.

Proenza, F. (2001). Telecenter sustainability: myths and opportunities. The journal of Development Communication 12(2): 14 .

Proenza, F., R. Bastidas-Buch, et al. (2001). Telecenters for Socioeconomic and Rural Development in Latin America and the Caribbean. Washington, D.C., IADB; FAO; ITU.

Saravia, M. (2003) Ideas para repensar la conectividad en áreas rurales. En: Otro lado de la brecha: perspectivas latinoamericanas y del Caribe ante la CMSI. (pp. 29-33). Caracas, RedISTIC.

Sen, A. K. (1999). Development as freedom. New York, Knopf.

Stoll, K. y M. Menou (2003). Basic principles of telecenter sustainability. Retrieved March 28, 2005 from http://www.tele-centros.org/CR/crsosten.php.

Thomas, A. (2000). Meanings and views of development. En: Poverty and development: into de 21 st century / ed. by T. Allen y A. Thomas. Oxford: Open University \& Oxford University Press, pp 22-48.

UNDP (2004). Human development report 2004: Cultural liberty in today's diverse world. New York, UNDP.

WCED (1987). Our common future. Oxford: Oxford University Press. 


\section{Anexo: Metodología de esta investigación}

El análisis presentado en este artículo se basa en la información recogida para la evaluación de medio término encargada al autor por la ONG promotora del proyecto (Centro Peruano de Estudios Sociales - CEPES http://www.cepes.org.pe) en febrero y marzo de 2005. Para la evaluación del proyecto se combinaron metodologías y herramientas cuantitativas y cualitativas ya que necesitábamos indicadores objetivamente verificables e información de contexto y a profundidad.

\section{Herramientas cuantitativas}

Se aplicaron dos tipos de encuesta: una a usuarios de riego y otra a usuarios de los telecentros. La encuesta a usuarios de riego tuvo como objetivos obtener información sobre situación económica de los agricultores del valle, saber qué mecanismos usan actualmente para tomar decisiones en las diversas etapas del ciclo agrícola, así como las principales fuentes de información en las cuales se apoyan estas decisiones, y el grado de conocimiento de los telecentros y su uso. Los objetivos de la encuesta a usuarios de telecentros fueron identificar las características del actual usuario del telecentro (edad, sexo, ocupación, etc.), la forma en que se enteró de la existencia del telecentro, los servicios que más usa $\mathrm{y}$, si ha consultado la página del SIA, qué información buscó y si ésta le parece útil y comprensible.

\section{Herramientas cualitativas}

Se realizaron entrevistas a profundidad a diferentes actores relacionados con el proyecto así como talleres participativos. Los actores entrevistados a profundidad fueron dirigentes agrarios, representantes de asociaciones de productores, "sectoristas"-encargados de la supervisión del flujo de agua de riego-, personal del proyecto, personal administrativo de la Junta de Usuarios, administradores de telecentros y un grupo de "agricultores tipo" de acuerdo a las diferencias típicas entre ellos en cuanto a edad, origen de la propiedad de la tierra, educación, afiliación a organizaciones y género. La selección de informantes maximizó la variedad con la intención de obtener mayor riqueza en la información recogida y reflejar la variedad del universo (Bauer \& Aarts, 2002). Se realizaron talleres participativos para obtener información cualitativa de dos grupos: hubo un taller general con los múltiples actores relacionados al proyecto y uno con administradores de los telecentros, los que permitieron evaluar el proyecto, conocer los cambios que los actores estaban identificando y graficar flujos de información.

\section{Límites de la investigación}

Este artículo ha evitado tratar temas tecnológicos. La evaluación hecha a principios de 2005 no incluyó al software ni a las percepciones sobre el software usado en los telecentros, tampoco evaluamos el uso de otras herramientas novedosas como la conexión WiFi entre los telecentros de las comisiones, el uso de terminales tontos para dar servicio al público, ni el uso del CMS (sistema de manejo de contenidos) Action Aplications de la APC para permitir que varios actores puedan contribuir con el sistema. Todas estas opciones han tenido distinto tipo de impactos en el proyecto, pero como no pudimos abarcar esto en el recojo de información es poco lo que podríamos decir. Creemos que el análisis de la influencia de la elección y uso de estas herramientas en la sostenibilidad tecnológica del sistema y de ésta en la sostenibilidad en general bien merecen otros estudios.

Este artículo tampoco ha estudiado la sostenibilidad financiera en sí misma, es decir en cuánto la venta de servicios por parte de los telecentros cubre los costos de operación y cuáles son sus estrategias para conseguir más fondos por esta vía. Creemos que los telecentros de Huaral están condenados a no ser sostenibles financieramente si siguen el esquema de cabina y anclan en la sola provisión de acceso a Internet como fuente de ingresos; esto porque en las localidades donde no hay mercado los ingresos no serán suficientes, en las que lo haya o se lo cree tarde o temprano un empresario local instalará una cabina que funcione con Windows (pirata, probablemente), explote a sus trabajadores y permita el acceso a pornografía, lo que en conjunto la hará más eficiente en la competencia por usuarios de Internet. Por ello el proyecto está desarrollando otras estrategias para conseguir fondos (como la venta de conexión inalámbrica a Internet en las comunidades a donde aún no llega el servicio), analizar la eficiencia de estas estrategias y como se acomodan a los objetivos del proyecto y contribuyen a la sostenibilidad general bien merece otro estudio.

Tampoco hemos abordado extensamente ni la sostenibilidad político/legal ni la organizacional, aunque en el análisis se las vea muy relacionadas a la sostenibilidad social o a su actor principal, la Junta de Usuarios de Huaral. Este caso aporta información para analizar la relación entre sostenibilidad social y política/legal pero para construir un artículo que revise esta relación sería importante recoger información de otros casos. En cuanto a la sostenibilidad organizacional, creemos que el análisis de ésta en Huaral sería especialmente interesante y que debería mirar con atención el componente de realización personal de los administradores de los telecentros así como de los voluntarios que trabajan con ellos. 
No hemos evaluado el efecto del uso de información agraria en la productividad o los ingresos de los agricultores. Consideramos que para que sea posible medir el impacto del sistema de información en la forma como toman decisiones los agricultores y la calidad de estas decisiones se necesita un tiempo mínimo después del inicio de la operatividad del sistema -y no de la sola conexión a Internet-, pues se necesita generar confianza en el agricultor y dar tiempo para que el agricultor ensaye con la información (Bossio, 2002) y con el sistema. Considerando que muchos ciclos agrícolas duran un año, este tiempo debería ser de 2 años cuando menos. 